\title{
On the effectiveness of time and date-based sun positioning solar collector in tropical climate: a case study in Northern Peninsular Malaysia
}

\begin{abstract}
This paper provides detailed information on the developed hardware and software for sun tracking mechanism and shows the effectiveness of utilizing time and date-based sun positioning solar collector system in tropical climate. The sun positioning system is based on the calculated azimuth and altitude at location E100 $11^{\prime}, \mathrm{N} 6^{\circ} 26^{\prime}$ in Northern Peninsular Malaysia where the climate is categorized as tropical climate. The system has two axes tracking with accuracy of $1^{\circ}$ controlled by a programmable logic controller (PLC). The field test has been done during a sunny and clear day, cloudy day and, heavy overcast and rainy day in which the results show that the improvement in the generated power of $91.97 \%$, $122.71 \%$ and $90.42 \%$, respectively, as compared with the fixed horizontal system.
\end{abstract}

Keyword: Photovoltaic system; Solar tracking; Two-axis PV system 